\title{
Nong Lam University students' perceptions of corporate social responsibility: A case study
}

\author{
Na Le
}

Faculty of Economics, Nong Lam University, Ho Chi Minh City, Vietnam

\begin{abstract}
ARTICLE INFO
Research Paper

Received: June 13, 2019

Revised: September 20, 2019

Accepted: September 30, 2019

\section{Keywords}

Behavior

Corporate social responsibility

Nong Lam University students

Perceptions
\end{abstract}

\section{Corresponding author}

Le Na

Email: lena@hcmuaf.edu.vn

\section{ABSTRACT}

This study was conducted to assess students' perceptions of corporate social responsibility (CSR) and the impacts of those perceptions on their thinking and behavior. Structural equation modelling (SEM) was used to examine the proposed correlations by analysing the data collected from 787 students at Nong Lam University. Results showed that there were positive correlations from relatives, social activity participation, demographics, and self-perception of students' perception of CSR. Besides, we also found that perception would positively affect students' thinking and behavior.

Cited as: Le, N. (2019). Nong Lam University students' perceptions of corporate social responsibility: A case study. The Journal of Agriculture and Development 18(5), 1-9. 


\title{
Nghiên cứu nhận thức của sinh viên Trường Đại học Nông Lâm Thành phố Hồ Chí Minh về trách nhiệm xã hội của doanh nghiệp
}

\author{
Lê $\mathrm{Na}$
}

Khoa Kinh Tế, Trường Đại Học Nông Lâm TP.HCM, TP. Hồ Chí Minh

\section{THÔNG TIN BÀI BÁO}

\section{Bài báo khoa học}

Ngày nhận: 13/06/2019

Ngày chỉnh sửa: 20/09/2019

Ngày chấp nhận: 30/09/2019

\section{Từ khóa}

Hành vi

Nhận thức

Sinh viên Trường Đại học Nông Lâm TP.HCM

Trách nhiệm xã hội của doanh nghiệp

\section{*Tác giả liên hệ}

Lê $\mathrm{Na}$

Email: lena@hcmuaf.edu.vn

\section{TÓM TẮT}

Nghiên cứu nhằm xem xét các yếu tố ảnh hưởng đến nhận thức của sinh viên về trách nhiệm xã hội của doanh nghiệp (CSR), và tác động của nhận thức đến ý nghĩ và hành vi của các sinh viên khảo sát. Mô hình cấu trúc (SEM) được sử dụng để kiểm định các mối quan hệ thông qua dữ liệu thu thập từ 787 sinh viên Trường Đại học Nông Lâm TP. Hồ Chí Minh. Kết quả nghiên cứu chỉ ra có sự tác động cùng chiều từ các biến: gia đình người thân, tham gia hoạt động phong trào, nhân khẩu học và tự nhận thức tới nhận thức về $\mathrm{CSR}$ của sinh viên. Mặt khác có sự tác động cùng chiều từ nhận thức của sinh viên về CSR đến ý nghĩ và hành vi của chính mình.

\section{1. Đặt Vấn Đề}

Trách nhiệm xã hội của doanh nghiệp (CSR) đã được nghiên cứu từ những năm 30 của thế kỷ hai mươi với những công trình nghiên cứu của Berle \& Means (1932), Luo \& Bhattacharya (2006) và Nguyen (2010). Trách nhiệm xã hội của doanh nghiệp là vấn đề quan trọng trong quản lý doanh nghiệp, nó tác động đến nhiều mặt khác nhau như kinh tế (economic responsibilities), pháp lý (legal responsibilities), đạo đức (ethical responsibilities) và từ thiện (philanthropic responsibilities) (Carroll, 1991). Nghiên cứu của Balabanis \& ctv. (1998) đã chỉ ra mối liên hệ giữa CSR và sự phát triển kinh tế trong hoạt động của doanh nghiệp, và các nghiên cứu khác cũng chỉ ra rằng CSR làm cho doanh nghiệp phát triển tốt hơn thông qua sự hài lòng của khách hàng, ảnh hưởng tới hành vi tiêu dùng sản phẩm, làm gia tăng giá trị cho doanh nghiệp (Luo \& Bhattacharya, 2006; Marin \& ctv., 2009). Nghiên cứu của Sweeney (2009) khẳng định CSR tác động trực tiếp đến hoạt động tài chính thông qua tiếp cận nguồn vốn, giúp thu hút và thúc đẩy sự phát triển doanh nghiệp và đặc biệt là giữ chân nhân viên. Còn Ali \& ctv. (2010) kết luận, CSR là yếu tố quan trọng để giữ chân khách hàng. Tóm lại, đã có nhiều nghiên cứu về CSR xuất phát từ doanh nghiệp hoặc chủ doanh nghiệp nhằm mục đích phát triển doanh nghiệp lâu dài.

Sinh viên là đối tượng được đào tạo bài bản, có kiến thức tốt và trình độ chuyên môn cao, vì được đào tạo cả kiến thức, kỹ năng và thái độ. Họ phần lớn là thanh niên, trẻ trung, nhiều hoài bảo khởi nghiệp, sẽ là những người lao động tương lai trong các doanh nghiệp có trách nhiệm xã hội và là trụ cột trong phát triển và bảo vệ đất nước. Vì vậy việc nghiên cứu nhận thức của sinh viên về CSR là rất quan trong. Sinh viên có nhận thức tốt về CSR khi ra trường làm việc sẽ phát triển xã hội, đất nước được tốt hơn. Đã có vài nghiên cứu về nhận thức về $\mathrm{CSR}$ của sinh viên (Fitzpatrick, 2013; Teixeira \& ctv., 2018) các nghiên cứu đã chỉ ra CSR ảnh hưởng bởi giới tính, trình độ, kinh 
nghiệm và tuổi tác. Burcea \& Marinescu (2011) đã nhấn mạnh tầm quan trọng của nhận thức về $\mathrm{CSR}$ của sinh viên tại cấp độ học thuật, tác động tới mức độ tham gia vào các hoạt động học thuật, tấp huấn, hoạt động cộng đồng. Nghiên cứu trong nước nhấn mạnh nhận thức về $\mathrm{CSR}$ của sinh viên gồm trách nhiệm pháp lý, đạo đức, kinh tế và từ thiện (Nguyen \& ctv., 2014). Vì vậy, thực hiện một nghiên cứu để đánh giá thực trạng và đo lường các yếu tố ảnh hưởng tới nhận thức về $\mathrm{CSR}$ của sinh viên là rất cần thiết, từ đó đề ra các giải pháp nhằm nâng cao nhận thức về CSR cho sinh viên.

\section{Cơ Sở Lý Luận và Phương Pháp Nghiên Cứu}

\subsection{Nhận thức về trách nhiệm xã hội của doanh nghiệp}

Theo Ủy ban cộng đồng Châu Âu, CSR là khái niệm theo đó các công ty tích hợp các mối quan tâm xã hội và môi trường trong hoạt động kinh doanh và trong sự tương tác của họ với các bên liên quan trên cơ sở tự nguyện (Dahlsrud, 2008). Hội đồng kinh doanh thế giới vì sự phát triển bền vững tuyên bố năm 2000, thì CRS là cam kết tiếp tục của doanh nghiệp để hành xử có đạo đức và đóng góp cho phát triển kinh tế, đồng thời cải thiện chất lượng cuộc sống của lực lượng lao động và gia đình của họ, cũng như cộng đồng địa phương và xã hội (Dahlsrud, 2008). Nhiều nghiên cứu đưa ra các yếu tố trách nhiệm xã hội khác nhau, nhưng hầu hết đều đồng nhất có 4 nhóm là trách nhiệm kinh tế; pháp lý; đạo đức và từ thiện.

Mặt khác, các nghiên cứu chỉ ra có nhiều yếu tố ảnh hưởng tới nhận thức CSR của sinh viên như tuổi tác, bằng cấp, giới tính và kinh nghiệm... Từ sự kế thừa các nghiên cứu (Carroll, 1991; Fitzpatrick, 2013; Teixeira \& ctv., 2018) và thảo luận, rút ra kết luận "có nhiều yếu tố ảnh hưởng tới nhận thức về CSR của sinh viên" có thể được chia làm 4 nhóm như sau.

Gia đình - người thân: Gia đình - thầy cô - bạn bè thường gọi "Gia đình - người thân", là tập hợp những cá nhân luôn bên cạnh dạy dỗ kiến thức, chia sẽ kỹ năng và truyền đạt kinh nghiệm cho sinh viên. Hằng ngày sinh viên chịu các tác động trực tiếp từ gia đình và thầy cô, cũng như ảnh hưởng trong quá trình giao lưu kết nối từ bạn bè. Vì vậy, nếu gia đình - người thân có ý thức trách nhiệm tốt về CSR sẽ ảnh hưởng tới nhận thức về $\mathrm{CSR}$ của sinh viên. Từ đó đặt ra giả thuyết H1: Gia đình - người thân ảnh hưởng tích cực tới nhận thức của sinh viên về CSR.

Hoạt động phong trào: Hoạt động phong trào là quá trình sinh viên tham gia vào hoạt động của Đoàn thanh niên, Hội sinh viên, các câu lại bộ, đội nhóm. Các hoạt động phong trào ngoài tạo ra sân chơi, rèn luyện sức khỏe, kỹ năng, còn giáo dục sinh viên sống có trách nhiệm hơn với công đồng và xã hội. Nên có giả thuyết H2: Tham gia hoạt động phong trào ảnh hưởng tích cực tới nhận thức về CSR của sinh viên.

Nhân khẩu học: Nhân khẩu học là các yếu tố như tuổi tác, giới tính, kinh nghiệm, học vấn. Nhiều nghiên cứu đã chỉ ra nhân khẩu học ảnh hưởng tới nhận thức CSR (Fitzpatrick, 2013; Teixeira \& ctv., 2018). Mỗi giai đoạn tuổi tác khác nhau thì nhận thức về CSR của con người sẽ khác nhau, nhưng theo hướng nhiều tuổi thì nhận thức càng tốt hơn. Nhận thức về CSR của nữ tốt hơn nam giới, người có học vấn cao sẽ nhận thức được CSR rất quan trọng, các nghiên cứu còn chỉ ra rằng khi CSR tốt, doanh nghiệp thường làm ăn hiệu quả hơn (Fitzpatrick, 2013; Teixeira \& ctv., 2018). Nên có giả thuyết H3: Nhân khẩu học ảnh hưởng tích cực tới nhận thức của sinh viên về CSR.

Tự nhận thức: Tự nhận thức là quá trình con người suy nghĩ, đánh giá các vấn đề bằng tư duy lập luận, kiến thức học tập và quan sát thông qua hoạt động sống. Là quá trình phát triển của não bộ, làm cho con người biết được đúng-sai, việc gì nên-không nên làm (Bem, 1967; Efron, 1969; Bem, 1972). Tự nhận thức đưa con người bước tới cấp độ nhận thức cao hơn, và ảnh hưởng đến suy nghĩ và hành vi. Nên có giả thuyết $\mathrm{H} 4$ : Tự nhận thức ảnh hưởng tích cực tới nhận thức về CSR của sinh viên

\subsection{1. Ý nghĩ}

Ý nghĩ là những suy nghĩ bên trong, nên tảng của thái độ mỗi cá nhân. Theo McShane \& Von Glinow (2003), thái độ là những phát biểu hay những đánh giá, phản ánh cảm thâyy như thế nào về một điều gì đó. Ý nghĩ, được thể hiện thông qua 3 thành phần của thái độ. Thành phần nhận thức, bao gồm ý kiến hoặc niềm tin. Thành phần ảnh hưởng, là cảm nhận hay cảm xúc. Thành phần hành vi, là chủ ý cư xử theo một cách nào đó với một người hay một việc gì đó. Trong tổ 
chức ý nghĩ, thái độ quan trọng vì nó ảnh hưởng tới hành vi. Theo Bui (2011), thái độ là những biểu đạt có tính đánh giá, liên quan đến các vật thể, con người và các sự kiện. Mặt khác, ý nghĩ và thái độ xuất phát từ nhận thức. Vì vậy có giả thuyết H5: Nhận thức của sinh viên về CSR ảnh hưởng tích cực đến ý nghĩ của chính mình.

\subsubsection{Hành vi}

Hành vi là hành động của con người trong chừng mực nào đó, khi và chỉ khi cá nhân hành động gắn với một ý nghĩa chủ quan. Hành vi là hành động hướng đến người khác, mang tính chủ quan, xuất phát từ suy nghĩ (Bailey \& Gayle, 2003). Động cơ, đạo đức sẽ quyết định tới hành vi và hành động của người đó. Thường người có ý nghĩ tốt sẽ có hành vi tốt (Le \& Nguyen, 2016). Nên có giả thuyết H6: Ý nghĩ của sinh viên về CSR ảnh hưởng tích cực tới hành vi của chính mình.

\subsection{Phương pháp nghiên cứu}

Nghiên cứu thực hiện phỏng vấn 787 sinh viên Trường Đại học Nông Lâm TP.HCM, vào tháng 12 năm 2018. Kết quả nam 43,10\%; nữ 56,90\%. Ở ký túc xá $42,60 \%$; thuê phòng $39,30 \%$; ở với gia đình $11,80 \%$ và ở với họ hàng $3,90 \%$. Sinh viên của mẫu khảo sát có $1,70 \%$ bộ môn Công nghệ hóa học; 4,10\% bộ môn Công nghệ sinh học; 4,30\% khoa Công nghệ thông tin; 9,30\% khoa Công nghệ thực phẩm; $17,30 \%$ khoa Chăn nuôi thú ý; 7,60\% khoa Cơ khí công nghệ; 16,50\% khoa Kinh tế; 2,40\% khoa Lâm nghiệp; 7,60\% khoa Môi trường tài nguyên; $2,30 \%$ khoa Ngoại ngữ sư phạm; 9,70\% khoa Nông học; 10,90\% khoa Quản lý đất đai và bất động sản và $6,40 \%$ khoa Thủy sản. Mẫu khảo sát khá cân đối về giới tính, chỗ ở và ngành học của các sinh viên, và đều đạt yêu cầu về cở mẫu, nên đủ độ tin cậy trong thống kê.

Các thang đo được kế thừa từ các nghiên cứu trước (Luo \& Bhattacharya, 2006; Sweeney, 2009; Teixeira \& ctv., 2018) và tổng hợp xây dựng thêm. Kết quả khảo sát được sử dụng để đánh giá và kiểm định mô hình thang đo, mô hình nghiên cứu cùng các giả thuyết nghiên cứu và cuối cùng dùng để so sánh mô hình cấu trúc đa nhóm. Thực hiện kiểm định qua 2 bước: Đánh giá độ tin cậy của các thang đo thông qua chỉ số Cronbach alpha $(0,95 \geq$ Cronbach alpha $\geq 0,6)$ (Nunnally \& Bernstein, 1994) và thực hiện phân tích nhân tố khám phá EFA (Exploratory Factor Analysis), thang đo được chấp nhận khi tổng phương sai trích $\geq 50 \%$ và hệ số $\mathrm{KMO}>0,5$ (Anderson \& Gerbing, 1988). Kiểm định mô hình nghiên cứu sử dụng CFA (Confirmatory Factor Analysis) phân tích yếu tố khẳng định để khẳng định độ tin cậy và độ giá trị thang đo, sử dụng mô hình cấu trúc SEM (Structural Equation Modellong) để ước lượng mô hình thang đo và mô hình cấu trúc, kiểm tra mối quan hệ phức hợp trong mô hình.

\section{Kết Quả và Thảo Luận}

\subsection{Thực trạng nhận thức về CSR của sinh viên}

Nhận định về giai đoạn hình thành ý thức trách nhiệm của con người, $34,40 \%$ sinh viên được hỏi cho rằng từ giai đoạn sinh ra đến 12 tuổi, và $44,00 \%$ cho rằng từ giai đoạn 13 đến 17 tuổi, và 19,60\% lại cho rằng từ giai đoạnh 18 đến 22 tuổi. Tóm lại, có $98,00 \%$ sinh viên được hỏi cho rằng ý thức trách nhiệm của con người được hình thành trong giai đoạn từ lúc sinh ra đến 22 tuổi.

Nhận định về nguồn gốc chủ yếu hình thành trách nhiệm của con người, $47,40 \%$ sinh viên cho rằng phải được đào tạo, $31,40 \%$ cho rằng có thể đào tạo được và $14,60 \%$ cho rằng do yếu tổ bẩm sinh.

Sinh viên nhận định yếu tố ảnh hướng tới sự hình thành ý thức trách nhiệm của con người, $31,40 \%$ cho rằng do tự ý thức được, $45,20 \%$ cho rằng do yếu tố giáo dục của gia đình; $5,50 \%$ cho rằng do thầy cô và bạn bè, $9,50 \%$ cho rằng do chương trình đạo tạo và $8,40 \%$ cho rằng do các yếu tố khác.

\section{2. Đo lường các yếu tố ảnh hưởng tởi nhận thức trách nhiệm xã hội của sinh viên}

\subsubsection{Kiểm định thang đo}

Kết quả phân tích nhân tố khám phá Cronbach alpha cho thấy, Cronbach alpha các biến độc lập và phụ thuộc đều > 0,6 cụ thể: Gia đình người thân GDNT $(0,667)$; Hoạt động phong trào HDPT $(0,741)$; Nhân khẩu học NKH $(0,783)$; Tự nhận thức TNT $(0,696)$; Nhận thức về CSR NT (0,748); Ý nghĩ YN $(0,838)$; Hành vi HV $(0,846)$ (Bảng 1). Các Alpha loại biến < Cronbach alpha biến tổng. Phân tích EFA cho trị số $\mathrm{KMO}=$ 0,848 và Tổng phương sai trích $=50,535 \%$. 
Bảng 1. Kết quả phân tích EFA, ma trận xoay

\begin{tabular}{|c|c|c|c|c|c|c|c|c|}
\hline \multirow{2}{*}{ Khái niệm } & \multirow{2}{*}{ Biến quan sát } & \multicolumn{7}{|c|}{ Yếu tố } \\
\hline & & 1 & 2 & 3 & 4 & 5 & 6 & 7 \\
\hline \multirow{3}{*}{ Gia đình - người thân } & GDNT1 & & & & & & & $\overline{0,510}$ \\
\hline & GDNT2 & & & & & & & 0,804 \\
\hline & GDNT3 & & & & & & & 0,598 \\
\hline \multirow{3}{*}{ Hoạt động phong trào } & HDPT1 & & & & & & 0,646 & \\
\hline & HDPT2 & & & & & & 0,756 & \\
\hline & HDPT3 & & & & & & 0,684 & \\
\hline \multirow{3}{*}{ Nhân khẩu học } & NKH1 & & & 0,635 & & & & \\
\hline & NKH2 & & & 0,791 & & & & \\
\hline & NKH3 & & & 0,739 & & & & \\
\hline \multirow{4}{*}{ Tự nhận thức } & TNT1 & & & & & 0,668 & & \\
\hline & TNT2 & & & & & 0,484 & & \\
\hline & TNT3 & & & & & 0,785 & & \\
\hline & TNT4 & & & & & 0,477 & & \\
\hline \multirow{4}{*}{ Nhận thức về CSR } & NTTN1 & & & & 0,642 & & & \\
\hline & NTTN2 & & & & 0,712 & & & \\
\hline & NTTN3 & & & & 0,579 & & & \\
\hline & NTTN4 & & & & 0,656 & & & \\
\hline \multirow{3}{*}{ Ý nghĩ } & YN1 & & 0,918 & & & & & \\
\hline & YN2 & & 0,715 & & & & & \\
\hline & YN3 & & 0,769 & & & & & \\
\hline \multirow{5}{*}{ Hành vi } & HV1 & 0,520 & & & & & & \\
\hline & HV2 & 0,648 & & & & & & \\
\hline & HV3 & 0,770 & & & & & & \\
\hline & HV4 & 0,826 & & & & & & \\
\hline & HV5 & 0,825 & & & & & & \\
\hline \multirow{3}{*}{\multicolumn{2}{|c|}{$\begin{array}{l}\text { Eigenvalues } \\
\text { Tổng phương sai trích }=50,535 \% \\
\text { Cronbach's Alpha }\end{array}$}} & 5,866 & 2,722 & 1,885 & 1,583 & 1,531 & 1,271 & 1,112 \\
\hline & & 21,535 & 9,162 & 5,646 & 4,661 & 4,153 & 3,027 & 2,351 \\
\hline & & 0,846 & 0,838 & 0,783 & 0,748 & 0,696 & 0,741 & 0,667 \\
\hline
\end{tabular}

Phân tích CFA áp dụng kết hợp với phương pháp ước lượng ML. Kết quả các biến độc lập, trung gian và phụ thuộc là các thang đo đơn hướng, nên ta có mô hình tới hạn với Chiquare $=537,544 ; \mathrm{df}=253 ;(P=0,000)$. Chỉ số $\mathrm{CMIN} / \mathrm{df}=2,125$ đạt yêu cầu cho độ tương thích, $(\mathrm{CFI}=0,949 ; \mathrm{TLI}=0,948 ; \mathrm{CFI}=0,957)$ và $\mathrm{RM}$ SEA $=0,038$ kết luận mô hình đạt độ tương thích với dữ liệu thị trường.

Kết quả CFA mô hình tới hạn khẳng định tính đơn hướng (do sự phù hợp của mô hình và không có tương quan giữa các sai số các biến quan sát) và giá trị hội tụ của 7 thang đo đơn hướng gồm Gia đình người thân (GDNT); Hoạt động phong trao (HDPT); Nhân khẩu học (NKH); Tự nhận thức (TNT); Nhận thức về CSR (NT); Ý nghĩ $(\mathrm{YN})$ và Hành vi $(\mathrm{HV})$ đều có trọng số $\left(\lambda_{\mathrm{i}}\right)$ khá cao (thấp nhất là TNT2 $=0,49$ ) và text $<0,05$. Kết luận các biến quan sát dùng để đo lường các khái niệm đơn hướng đều đạt giá trị hội tụ. Ngoài ra, độ tin cậy tổng hợp thang đo các khái niệm đơn hướng đều đạt giá trị cao (thấp nhất là GDNT $=0,680$ ) và tổng phương sai trích đều khá lớn (bé nhất là $\mathrm{TNT}=0,377$ ) khẳng định các thang đo là đơn hướng và đạt yêu cầu về độ giá trị và độ tin cậy trong nghiên cứu (Bảng 2). Các hệ số tương quan giữa các khái niệm nghiên cứu đều $<1 ; P=0,000$ và hệ số tới hạn $\mathrm{C} . \mathrm{R}>$ 2 , nói cách khác, các khái niệm nghiên cứu đạt giá trị phân biệt (Bảng 3).

\subsubsection{Kiểm định mô hình nghiên cứu và các giả thuyết}

Kết quả phân tích SEM có $\mathrm{df}=260$; Chi-quare $=795,315(P=0,000) ; \mathrm{CMIN} / \mathrm{df}=3,059$ và $(\mathrm{GFI}=0,927 ; \mathrm{TLI}=0,905 ; \mathrm{CFI}=0,918$ và $\mathrm{RM}-$ SEA $=0,051)$ đều phù hợp. Kết luận mô hình nghiên cứu thích hợp với dữ liệu thu thập từ thị 
Bảng 2. Tóm tắt kết quả kiểm định thang đo các khái niệm đơn hướng

\begin{tabular}{lccccc}
\hline Thành phần & $\begin{array}{c}\text { Số biến } \\
\text { quan } \\
\text { sát }\end{array}$ & $\begin{array}{c}\text { Độ tin } \\
\text { cậy tổng } \\
\text { hợp }\end{array}$ & $\begin{array}{c}\text { Tổng } \\
\text { phương } \\
\text { sai trích }\end{array}$ & $\begin{array}{c}\text { Trung } \\
\text { bình hệ } \\
\text { số tải }\end{array}$ & $\begin{array}{c}\text { Giá trị hội } \\
\text { tụ và phân } \\
\text { biệt }\end{array}$ \\
\hline Gia đình người thân (GDNT) & 3 & 0,680 & 0,427 & 0,638 & Thỏa mãn \\
Hoạt động phong trào (HDPT) & 3 & 0,743 & 0,492 & 0,701 & Thỏa mãn \\
Nhân khẩu học (NKH) & 3 & 0,785 & 0,550 & 0,741 & Thỏa mãn \\
Tự nhận thức (TNT) & 4 & 0,704 & 0,377 & 0,608 & Thỏa mãn \\
Nhận thức về CSR (NT) & 4 & 0,749 & 0,428 & 0,654 & Thỏa mãn \\
Ý nghĩ (YN) & 3 & 0,847 & 0,650 & 0,805 & Thỏa mãn \\
Hành vi (HV) & 5 & 0,832 & 0,500 & 0,704 & Thỏa mãn \\
\hline
\end{tabular}

Bảng 3. Kết quả kiểm định giá trị phân biệt giữa các khái niệm nghiên cứu

\begin{tabular}{|c|c|c|c|c|c|c|}
\hline \multicolumn{3}{|c|}{ Mối quan hệ } & $\bar{E}$ & S.E & C.R & $\bar{P}$ \\
\hline GDNT & $\leftrightarrow$ & HDPT & 0,209 & 0,035 & 22,663 & 0.000 \\
\hline HDPT & $\leftrightarrow$ & NKH & 0,107 & 0,035 & 25,164 & 0,000 \\
\hline NKH & $\leftrightarrow$ & TNT & 0,001 & 0,036 & 27,990 & 0,000 \\
\hline GDNT & $\leftrightarrow$ & NKH & 0,124 & 0,035 & 24,735 & 0,000 \\
\hline HDPT & $\leftrightarrow$ & TNT & 0,129 & 0,035 & 24,609 & 0,000 \\
\hline GDNT & $\leftrightarrow$ & TNT & 0,104 & 0,035 & 25,241 & 0,000 \\
\hline GDNT & $\leftrightarrow$ & NT & 0,154 & 0,035 & 23,989 & 0,000 \\
\hline GDNT & $\leftrightarrow$ & YN & 0,099 & 0,036 & 25,369 & 0,000 \\
\hline GDNT & $\leftrightarrow$ & $\mathrm{HV}$ & 0,093 & 0,036 & 25,523 & 0,000 \\
\hline HDPT & $\leftrightarrow$ & NT & 0,153 & 0,035 & 24,014 & 0,000 \\
\hline HDPT & $\leftrightarrow$ & YN & 0,134 & 0,035 & 24,484 & 0,000 \\
\hline HDPT & $\leftrightarrow$ & HV & 0,157 & 0,035 & 23,916 & 0,000 \\
\hline NKH & $\leftrightarrow$ & NT & 0,216 & 0,035 & 22,497 & 0,000 \\
\hline $\mathrm{NKH}$ & $\leftrightarrow$ & YN & 0,250 & 0,035 & 21,703 & 0,000 \\
\hline NKH & $\leftrightarrow$ & $\mathrm{HV}$ & 0,075 & 0,036 & 25,990 & 0,000 \\
\hline TNT & $\leftrightarrow$ & NT & 0,127 & 0,035 & 24,659 & 0,000 \\
\hline TNT & $\leftrightarrow$ & YN & 0,029 & 0,036 & 27,217 & 0,000 \\
\hline TNT & $\leftrightarrow$ & $\mathrm{HV}$ & 0,107 & 0,035 & 25,164 & 0,000 \\
\hline NT & $\leftrightarrow$ & YN & 0,186 & 0,035 & 23,212 & 0,000 \\
\hline NT & $\leftrightarrow$ & $\mathrm{HV}$ & 0,150 & 0,035 & 24,088 & 0,000 \\
\hline YN & $\leftrightarrow$ & HV & 0,082 & 0,036 & 25,807 & 0,000 \\
\hline
\end{tabular}

trường.

Ước lượng $\mathrm{R}_{\text {(Nhận thức) }}^{2}=0,464$, biến Nhận thức của sinh viên về CSR (NT) được giải thích bởi 4 yếu tố Gia đình - người thân (GDNT); Hoạt động phong trào (HDPT); Nhân khẩu học (NKH) và Tự nhận thức (TNT). $\mathrm{R}_{(\text {Ý nghĩa) }}^{2}=0,205$, biến Ý nghĩ (YN) được giải thích bởi yếu tố Nhận thức về $\mathrm{CSR}(\mathrm{NT})$ của sinh viên. $\mathrm{R}_{(\text {Hành vi) }}^{2}=0,031$, biến Hành vi (HV) được giải thích bởi nhân tố Ý nghĩ (YN) của sinh viên.

Kiểm định Bootstrap với số lượng mẫu lặp lại $\mathrm{N}=1000$, kết quả < 0,001 kết luận các ước lượng trong mô hình là tin cậy. Hơn thế, kết quả SEM ước lượng mô hình chuẩn hóa cho thấy các giả thuyết $\mathrm{H} 1, \mathrm{H} 2, \mathrm{H} 3, \mathrm{H} 4, \mathrm{H} 5$ và $\mathrm{H} 6$ có $P=0,000$ nên được chấp nhận (Bảng 4). So sánh sự khác biệt của mô hình nghiên cứu theo biến định tính nam - nữ. Kết quả không có sự khác biệt theo nhóm kiểm định (Bảng 5).

\subsection{Thảo luận}

Kết quả nghiên cứu chỉ ra 4 nhân tố ảnh hưởng tới nhận thức về $\mathrm{CSR}$ của sinh viên với $\mathrm{R}^{2}=$ 46,4\%. Vậy để nâng cao nhận thức CSR cho sinh viên cân thực hiện những biện pháp sau. 
Bảng 4. Quan hệ giữa các khái niệm trong mô hình

\begin{tabular}{ccccccccc}
\hline Giả thuyết & \multicolumn{2}{c}{ Mối quan hệ } & Estimate & S.E. & C.R. & & Kiểm định \\
\hline H1 & GDNT & $\leftrightarrow$ & NT & 0,120 & 0,033 & 26,283 & 0,000 & Chấp nhận \\
H2 & HDPT & $\leftrightarrow$ & NT & 0,130 & 0,033 & 26,133 & 0,000 & Chấp nhận \\
H3 & NKH & $\leftrightarrow$ & NT & 0,438 & 0,027 & 21,004 & 0,000 & Châ̂p nhận \\
H4 & TNT & $\leftrightarrow$ & NT & 0,347 & 0,029 & 22,641 & 0,000 & Chấp nhận \\
H5 & NT & $\leftrightarrow$ & YN & 0,452 & 0,026 & 20,741 & 0,000 & Chấp nhận \\
H6 & YN & $\leftrightarrow$ & HV & 0,177 & 0,032 & 25,418 & 0,000 & Chấp nhận \\
\hline
\end{tabular}

Bảng 5. Sự khác biệt theo giới tính

\begin{tabular}{ccccc}
\hline Biến kiểm định & Mô hình so sánh & Chi-square & Df & $P$ \\
\hline \multirow{2}{*}{ Giới tính } & Bất biến từng phần & 1141,446 & 520 & 0,000 \\
(Nam-Nữ) & Khả biến & 1151,857 & 526 & 0,000 \\
& Giá trị khác biệt & 10,411 & 6 & 0,108 \\
\hline
\end{tabular}

\subsubsection{Biện pháp rút ra từ phân tích các biến nhân khẩu học cho sinh viên}

Biến Nhân khẩu học (NKH) tác động đến biến Nhận thức về $\mathrm{CSR}(\mathrm{NT})$ của sinh viên với hệ số 0,438 , kết quả này cũng thống nhất với nghiên cứu của Fitzpatrick (2013) và Teixeira \& ctv. (2018). Kết quả 3 thang đo của biến Nhân khẩu học là (Giới tính) NKH2 $=0,786$ với $P=0,000$; (Vùng miền) $\mathrm{NKH} 3=0,723$ với $P=0,000$; và (Trình độ, bằng cấp) $\mathrm{NKH} 1=0,713$ với $P=$ 0,000 . Để nhận thức về CSR tăng cần thúc dẩy các giá trị nhân khẩu học cho sinh viên. Yếu tố trình độ, bằng cấp có thể cải thiện được trong ngắn và trung hạn. Sinh viên có trình độ sẽ biêt được nội dung và tầm quan trọng của CSR. Việc nâng cao trình độ cho sinh viên hết sức quan trọng, sinh viên cần được trang bị các kiến thức chuyên ngành và các kiến thức về tự nhiên, xã hội, đạo đức, pháp lý, trách nhiệm xã hội,... để ra trường làm tốt các công việc. Mặt khác, việc da dạng vùng miền giúp sinh viên có cơ hội giao lưu và thích nghi với nhiều vùng văn hóa, học hỏi được tính ưu việt của các vùng miền giúp phát triển bản thân và nhận thức được tốt hơn về CSR.

\subsubsection{Nâng cao khả năng tự nhận thức cho sinh viên}

Biến Tự nhận thức (TNT) ảnh hưởng tới biến Nhận thức về CSR (NT) của sinh viên với hệ số là 0,347 . Biến TNT được đo bằng 4 thang đo với hệ số tác động là TNT1 Ý thức tự giác $(0,612)$; TNT2 Môi trường sống $(0,490)$; TNT3 Chịu trách nhiệm về đạo đức $(0,728)$ và TNT4 Ý thức trách nhiệm $(0,593)$ đều đạt ý nghĩa thống kê. Vì vậy, dể tăng cường nhận thức của sinh viên về $\mathrm{CSR}$ cần nâng cao khả năng tự nhận thức. Khi sinh viên tự nhận thức được CSR sẽ phát huy được tính đọc lập trong suy nghĩ và hành động. Để làm được việc này, chương trình học cần cung cấp các học phần về tư duy, pháp luật, văn hóa doanh nghiệp, hơn nữa là các học phần liên quan tới CSR,... Việc bổ sung các học phần về CSR vào chương trình đào tạo các trường đại học trên thế giới đã thực hiện từ khá sớm, không những ở các chương trình sau đại học mà ngay cả chương trình đào tạo đại học (Thøger Christensen \& ctv., 2008). Thông qua chương trình, dạy cho sinh viên tư duy độc lập, phương pháp phản biện logic, kiến thức về đạo đức, trách nhiệm. Từ đó sinh viên sẽ nhận thức được những gì là đúng-sai, sẽ tự nâng cao được ý thức tự giác, tự chịu trách nhiệm, nâng cao hơn khả năng nhận thức về CSR của mình.

\subsubsection{Tăng cường hoạt động phong trào cho sinh viên}

Biến Hoạt động phong trào (HDPT) tác động tới biến Nhận thức của sinh viên về $\mathrm{CSR}(\mathrm{NT})$ là 0,130. Biến HDPT được đo lường bằng 3 thang đo với kết quả là HDPT1 Tham gia hoạt động Đoàn-Hội $(0,674)$; HDPT2 Tham gia hoạt dộng tình nguyện $(0,719)$ và HDPT3 Tham gia hoạt động các câu lạc bộ $(0,710)$ đều đạt ý nghĩ thống kê. Để tăng nhận thức về CSR cho sinh viên cần tăng cường hoạt động phong trào. Thông qua trải nghiệm các hoạt động phong trào sinh viên sẽ nhận thức tốt hơn tầm quan trọng của CSR. (Hiện một số hoạt dộng phong trào giáo dục CSR cho sinh viên rất tốt như: Mùa hè xanh 
tình nguyện; Xuân tình nguyện; Trung thu cho em; Ngày môi trường thế giới $5 / 6$; Hiến máu tình nguyện,...). Vậy để nâng cao hơn nhận thức về CSR cho sinh viên cần có thêm các chương trình có tính liên tục, cụ thể như "Ngày chủ nhật xanh" đề xuất mỗi sinh viên thực hiện 1 ngày chủ nhật xanh trong mỗi học kỳ, nhà trường cho sinh viên đăng ký online và thực hiện, sẽ đạt các mục tiêu (1) Gắn trách nhiệm của sinh viên với môi trường sống; (2) Tạo khuôn viên trường xanh sạch đẹp; (3) giảm chi phí nhờ sức lao động của sinh viên; (4) Có thể tạo hiệu ứng tích cực trong xã hội, việc áp dụng giao cho Đoàn Thanh niên, Hội Sinh viên lên kế hoạch và thực hiện.

\subsubsection{Nâng cao CSR cho Gia đình - người thân}

Biến Gia đình - người thân (GDNT) tác động lên biến Nhận thức về $\mathrm{CSR}(\mathrm{NT})$ của sinh viên là 0,120 . Biến GDNT được đo bằng 3 thang đo với kết quả là GDNT1 Bạn bè (0,523); GDNT2 Thầy cô $(0,838)$ và GDNT3 Gia đình $(0,552)$ dều đạt ý nghĩ thống kê. Vậy muốn tăng cường nhận thức về CSR cho sinh viên cần nâng cao nhận thức CSR của gia đình và người thân. Tác động của thang đo GDNT2 là vượt trội so với hai thang đo còn lại, nên ảnh hưởng của thầy cô lên biến Gia đình - người thân là rất lớn. Vậy Thầy cô cần làm gương để sinh viên noi theo, như trong công việc cần hết lòng thương yêu giúp đỡ học trò, có trách nhiệm với chất lượng đào tạo, trong đánh giá cần công bằng, chuẩn mực... tạo nên đạo đức nghề nghiệp tốt và lan tỏa cho sinh viên. Mặt khác, để con cháu cư thể là sinh viên có CSR tốt, trước tiên phụ huynh cần gương mẫu, con cháu sẽ noi theo. Để nâng cao kết quả giáo dục sinh viên cần có sự kết hợp chặt chẽ giữa nhà trường và gia đình, hai bên cần thường xuyên trao đổi về tình hình học tập và phát triển của sinh viên.

\subsubsection{Hàm ý góp phần tác động hành vi của sinh viên}

Nhận thức về CSR sẽ ảnh hưởng tới ý nghĩ và hành vi của sinh viên, kết quả nghiên cứu đã chỉ ra Nhận thức về CSR (NT) tác động đến Ý nghĩ (YN) là 0,452 và Ý nghĩ (YN) tác động đến Hành vi $(\mathrm{HV})$ là 0,177 . Vì vậy để ý nghĩ và hành vi được tốt cần thực hiện đào tạo cân bằng giữa "kiến thức"; "kỹ năng"; "thái độ". Từ trước tới nay các trường đại học Việt Nam nói chung và Trường Đai học Nông Lâm TP. Hồ Chí Minh nói riêng đào tạo nặng về hàn lâm, nay ngoài kiến thức chuyên ngành, cần trang bị cho sinh viên về kỹ năng (tư duy; thu thập đánh giá thông tin; giao tiếp... ) và thái độ, vì thường thái độ tốt sẽ dẫn đến hành vi tốt. Để làm được việc này, chương trình đào tạo cần được bổ sung thêm tín chỉ về kỹ năng và thái độ.

\section{Kết Luận và Kiến Nghị}

\subsection{Kết luận}

Kết quả nghiên cứu đóng góp 2 mặt, là phương pháp nghiên cứu và giá trị sử dụng.

Về mặt phương pháp: Nghiên cứu đã bổ sung thêm các thang đo của các biên nghiên cứu: gia đình người thân, hoạt động phong trào, nhân khẩu học, tự nhận thức, nhận thức về CSR của sinh viên, ý thức và hành vi. Các thang đo đều đạt được độ tin cậy và giá trị phân biệt, đã mở thêm hướng đo lường nhận thức về CSR.

Về mặt giá trị nội dung: Từ kết quả tổng hợp 787 quan sát, nghiên cứu đã chỉ ra được giai đoạn hình thành và các yếu tố ảnh hưởng tới nhận thức về $\mathrm{CSR}$ của sinh viên. Bên cạnh đó nghiên cứu còn chỉ ra được các biến và thang đo dùng để do lường nhận thức về $\mathrm{CSR}$ của sinh viên là gia đình người thân, hoạt động phong trào, nhân khẩu học và tự nhận thức. Mặt khác kết quả còn chỉ ra có mối liên quan từ nhận thức về CSR tới ý nghĩ và hành vi. Từ đó đã đề ra được một số giải pháp nhằm nâng cao nhận thức về $\mathrm{CSR}$ cho sinh viên có ý nghĩa thực tiễn cho các nhà quản trị đại học Việt Nam nói chung và Trường Đại học Nông Lâm TP. Hồ Chí Minh là rất cần thiết.

\subsection{Kiến nghị}

Tương tự các nghiên cứu khác, khi sử dụng kết quả cần lưu ý.

Nghiên cứu chỉ xem xét 4 yếu tố tác động đến Nhận thức về CSR của sinh viên (gia đình - người thân; hoạt động phong trào; nhân khẩu học và tự nhận thức), nhưng trong thực tế có nhiều yếu tố khác tác động như chương trình đào tạo, văn hóa, tín ngưỡng,...

Để có giải pháp tốt hơn cho các nhà quản trị đại học, việc thu thập số liệu nên được phủ khắp TP. Hồ Chí Minh hoặc cả nước khi đó kết quả sẽ khách quan hơn. 


\section{Tài Liệu Tham Khảo (References)}

Ali, I., Rehman, K. U., Yilmaz, A. K., Nazir, S., \& Ali, J. F. (2010). Effects of corporate social responsibility on consumer retention in the cellular industry of Pakistan. African Journal of Business Management 4(4), 475485.

Anderson, J. C., \& Gerbing, D. W. (1988). Structural equation modelling in practice: A review and recommended two-step approach. Psychological Bulletin $103(3), 411$.

Bailey, G., \& Gayle, N. (2003). Social theory: Essential readings. Toronto, Canada: Oxford University Press.

Balabanis, G., Phillips, H. C., \& Lyall, J. (1998). Corporate social responsibility and economic performance in the top British companies: are they linked? European Business Review 98(1), 25-44.

Bem, D. J. (1967). Self-perception: An alternative interpretation of cognitive dissonance phenomena. Psychological Review 74(3), 183.

Bem, D. J. (1972). Self-perception theory. In Advances in experimental social psychology (Vol. 6, 1-62). Massachusetts, USA: Academic Press.

Berle, A., \& Means, G. (1932). Private property and the modern corporation. New York, USA: Macmillan.

Burcea, M., \& Marinescu, P. (2011). Students' Perceptions on Corporate Social Responsibility at the Academic Level. Case Study: The Faculty of Administration and Business, University of Bucharest. Amfiteatru Economic Journal 13(29), 207-220.

Bui, T. A., \& Pham, H. T. (2011). Organizational Behavioral Curriculum (1 ${ }^{\text {st }}$ ed.). Ha Noi, Vietnam: National Economics University Press.

Carroll, A. B. (1991). The pyramid of corporate social responsibility: Toward the moral management of organizational stakeholders. Business Horizons 34(4), 39-48.

Dahlsrud, A. (2008). How corporate social responsibility is defined: an analysis of 37 definitions. Corporate Social Responsibility and Environmental Management 15(1), 1-13.

Efron, R. (1969). What is perception? In Proceedings of the Boston Colloquium for the Philosophy of Science 1966/1968 (137-173). Dordrecht, Netherlands: Springer.
Fitzpatrick, J. (2013). Business students' perceptions of corporate social responsibility. College Student Journal 47(1), 86-95.

Le, N., \& Nguyen, T. T. M. (2016). University personality, external prestige and university reputation on supporting the attitude of university student. Journal of Agricultural Science and Technology 2, 106-114.

Luo, X., \& Bhattacharya, C. B. (2006). Corporate social responsibility, customer satisfaction, and market value. Journal of Marketing 70(4), 1-18.

Marin, L., Ruiz, S., \& Rubio, A. (2009). The role of identity salience in the effects of corporate social responsibility on consumer behavior. Journal of Business Ethics 84(1), 65-78.

McShane, S. L., \& Von Glinow, M. A. (2003). The employment relationship and career dynamics. Organizational behavior: Emerging realities for the workplace revolution (International ed., 552-555). New York, America: McGraw-Hill Education.

Nguyen, T. N. (2010). Linking human resource management with CSR. Ha Noi National University Journal of Science, Economics and Business (26), 232-238.

Nguyen, H. D., Tran, K. T., \& Le, X. T. T. (2014). CSR from the perception of university students. Journal of Science, Ho Chi Minh City Open University 5(38), 2840 .

Nunnally, J. C., \& Bernstein, I. H. (1994). Psychological theory. New York, USA: MacGraw-Hill.

Sweeney, L. (2009). A study of the current practice of corporate social responsibility (CSR) and an examination of the relationship between CSR and financial performance using structural equation modelling (SEM). (Unpublished doctoral dissertation). Technological University, Dublin, Ireland.

Teixeira, A., Ferreira, M. R., Correia, A., \& Lima, V. (2018). Students' perceptions of corporate social responsibility: evidence from a Portuguese higher education institution. International Review on Public and Nonprofit Marketing 15(2), 235-252.

Thøger Christensen, L., Fuat Frrat, A., \& Torp, S. (2008). The organisation of integrated communications: toward flexible integration. European Journal of Marketing 42(3/4), 423-452. 